Sie stimmen, wie aus der Abb. ersichtlich ist, gut mit den Meßwerten nach STEIN ${ }^{2}$ überein.

Als weiteres Beispiel soll die spontane Spaltung von $\mathrm{Cf}^{252}$ diskutiert werden. Zunächst berechnen wir (nach der Methode von Anm. ${ }^{1}$ ) aus der Spaltungswahrscheinlichkeit den Wert des Kernkraftpotentials $V_{\mathrm{K}}$ für ein festes $s$ in Abhängigkeit von $m$ (Abb. 2) und daraus

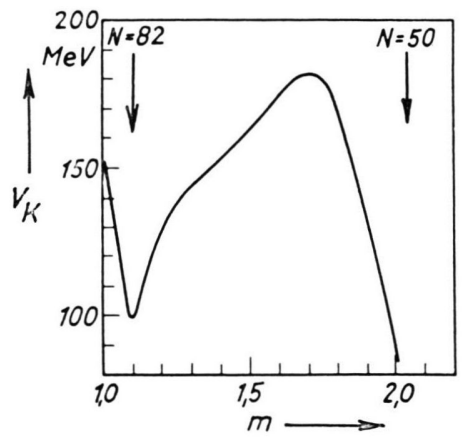

Abb. 2. Kernkraftwechselwirkung $V_{\mathrm{K}}$ für $s=14,0 \cdot 10^{-13} \mathrm{~cm}$ bei der spontanen Spaltung von $\mathrm{Cf}^{252}$ als Funktion des Massenverhältnisses (nach Anm. ${ }^{1}$ aus der Massenverteilung berechnet).

(ebenfalls nach Anm. ${ }^{1}$ ) die Deformation eines Bruchstückes in Abhängigkeit von seiner Neutronenzahl (Abb. 3). In beiden Kurven sind die „magischen Effekte" (Minima für den Fall, daß eines der Bruchstücke bezüglich seiner Neutronenzahl magisch ist $[N=50$ oder $N=82]$ ) deutlich erkennbar, wodurch die Überlegungen von Anm. ${ }^{1}$ eine weitere Stütze erfahren. Der aus der $\alpha^{2}$-Kurve der Abb. 3 nach Gl. (4) (mit $R_{1}{ }^{0}+R_{2}{ }^{0}$ $=11,9 \cdot 10^{-13} \mathrm{~cm}$ und $\lambda=40,71 \mathrm{MeV}$ ) folgende $T$.Ver-

2 W. E. Stein, Phys. Rev. 108, 94 [1957].

3 J. S. Fraser u. J. C. D. Milton, Proceedings of the Second

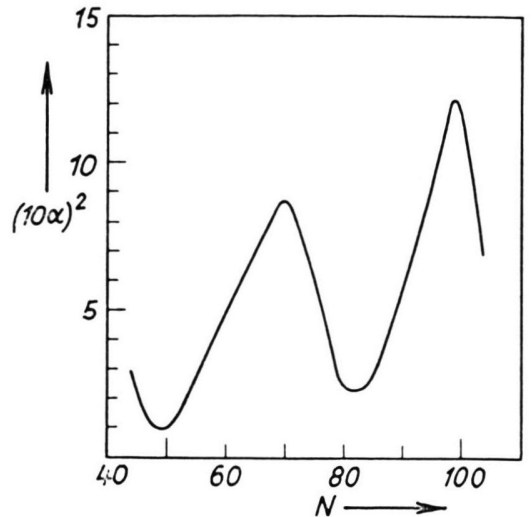

Abb. 3. Aus den $V_{\mathrm{K}}$-Werten der Abb. 2 nach Anm. ${ }^{1}$ berech nete Deformation eines Bruchstücks in Abhängigkeit von seiner Neutronenzahl $N$ bei der spontanen Spaltung von $\mathrm{Cf}^{252}$.

lauf ist in Abb. 1 mit eingezeichnet; die Úbereinstimmung mit den experimentellen Daten nach Fraser und Milton ${ }^{3}$ ist auch hier sehr gut.

Wir können somit feststellen, daß unsere Vorstellung der je nach der Schalenstruktur verschieden stark deformierten Bruchstücke sowohl zu einer Erklärung der Spaltungsasymmetrie (Anm. ${ }^{1}$ ) als auch des Verlaufs der kinetischen Energie $T$ der Fragmente führt. Damit erscheint der Versuch erfolgreich, die wesentlichen Charakteristika des Kernspaltungsprozesses als Folge des magischen Effektes in der Größe der Deformation der Bruchstücke zu verstehen.

Über die hier mitgeteilten Untersuchungen wird in Kürze an anderer Stelle ausführlicher berichtet werden.

United Nations International Conference on the Peaceful Uses of Atomic Energy, Geneva, P/199 [1958].

\section{Radiometrische Sedimentationsanalyse}

Von R. Lindner, J. O. Liljenzin und H. Reinhardt

Kernchemisches Institut der Technischen Hochschule Göteborg (Z. Naturforschg. 15 a, 1110-1112 [1960] ; eingeg. am 30. September 1960)

Bei Untersuchungen am hiesigen Institut (Diffusion von Spaltprodukten in Uranverbindungen ${ }^{1}$; Bestimmung der Größe von großen Radiokolloidteilchen ${ }^{2}$ ) ergab sich die Notwendigkeit, die Korngröße (oder die Dichte) kleiner Mengen $\alpha$-strahlender fester Stoffe zu bestimmen.

Der Gedanke lag nahe, die geringe Reichweite (max. $0,1 \mathrm{~mm}$ in Wasser) von $\alpha$-Strahlen zu einer „radiometrischen“ Sedimentationsmethode auszunutzen. Im

1 R. Lindner u. Hu. Matzke, Z. Naturforschg. 14 a, 1074 [1959]. vorliegenden Fall wird folgendes Prinzip verwendet: Bei Versuchsbeginn befindet sich das $\alpha$-strahlende Material aufgeschlämmt und homogen verteilt in einer Flüssigkeitssäule, um dann durch Sedimentation auf den Boden zu sinken und dort mit geeigneten Detektoren gemessen zu werden. Am einfachsten ist die direkte Befestigung des Sedimentationszylinders auf dem Endfenster eines Proportionalzählrohres, dessen Betriebsspannung so gewählt wird, daß nur $\alpha$-Strahlen registriert werden. Die Versuchsanordnung ist schematisch in Abb. 1 wiedergegeben.

Die Auswertung der Versuche basiert auf der Stokesschen Gleichung in der Form

$$
d=141 \sqrt{\frac{n h}{(\varrho-\varrho \mathrm{Fl}) t},},
$$

2 R. Lindner, J. O. Liljenzin u. H. Reinhardt, Z. Elektrochem. Ber. Bunsenges. physik. Chem., im Druck. 


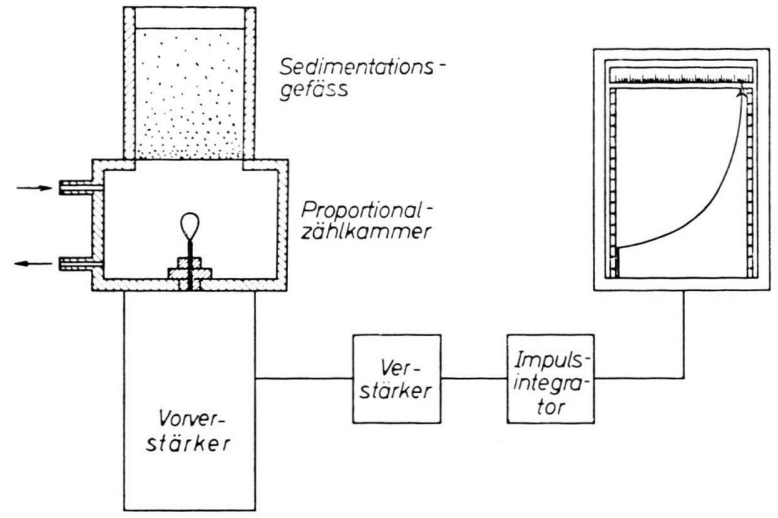

Abb. 1. Apparatur zur Messung der Sedimentation $a$-strahlender Festkörper (schematisch).

wobei $d$ die Kantenlänge eines kubischen Körpers in $\mu$, $\eta$ die Viskosität der Flüssigkiet in $\mathrm{g} \cdot \mathrm{sec} \cdot \mathrm{cm}^{-2}, h$ die Höhe der Flüssigkeitssäule in $\mathrm{cm}, \varrho_{\mathrm{Fl}}$ die Dichte der Flüssigkeit und $t$ die maximale Fallzeit in Minuten bedeuten.

Die einfachste Ermittlung einer Korngrößenverteilung geschieht analog dem Verfahren bei der Sedimentationswaage $^{3}$ folgendermaßen:

Mittels Registrierung der Radioaktivität $J$ wird die sich am Boden des Zylinders ansammelnde relative Stoff- menge $P$ als Funktion der Zeit bestimmt („Sedimentationskurve"; Abb. 2 a). An diese Kurve wird eine geeignete Anzahl Tangenten gelegt und die Werte ihrer Schnittpunkte $D$ mit der Ordinate gegen die zugehörigen Zeiten als neue Kurve („Durchgangskennlinie“) aufgetragen (Abb. 2 b). Es empfiehlt sich, die Durchgangskennlinie in einem neuen Diagramm aufzuzeich nen, wobei auf der Abszisse die nach der obigen Gleichung zu berechnenden Korngrößen aufgetragen werden. Aus diesem Diagramm (nicht wiedergegeben) werden die zu zweckmäßig gewählten Korngrößenintervallen gehörenden Ordinatendifferenzen abgelesen. Die so erhaltenen Werte werden gegen die Korngröße aufgetragen (Aktivitätsverteilungskurve, nicht wiedergegeben). Unter Berücksichtigung der Art der radioaktiven Indizierung (s. w. unten) wird die Häufigkeitsverteilungskurve (Abb. 2 c) berechnet. - Der Grundsatz dieser Auswertung ist schon von ODÉN ${ }^{3}$ angegeben worden: Die Sedimentationsfunktion $P(t)$ wird dort zweimal nach $t$ abgeleitet.

In vorliegendem Fall wird dagegen zunächst die Funktion

$$
D(t)=P(t)-t \frac{\mathrm{d} P(t)}{\mathrm{d} t}
$$

eingeführt (entsprechend der Durchgangskennlinie); nach einer weiteren Ableitung:

$$
\frac{\mathrm{d} D(t)}{\mathrm{d} t}=-t \frac{\mathrm{d}^{2} P(t)}{\mathrm{d} t^{2}}
$$

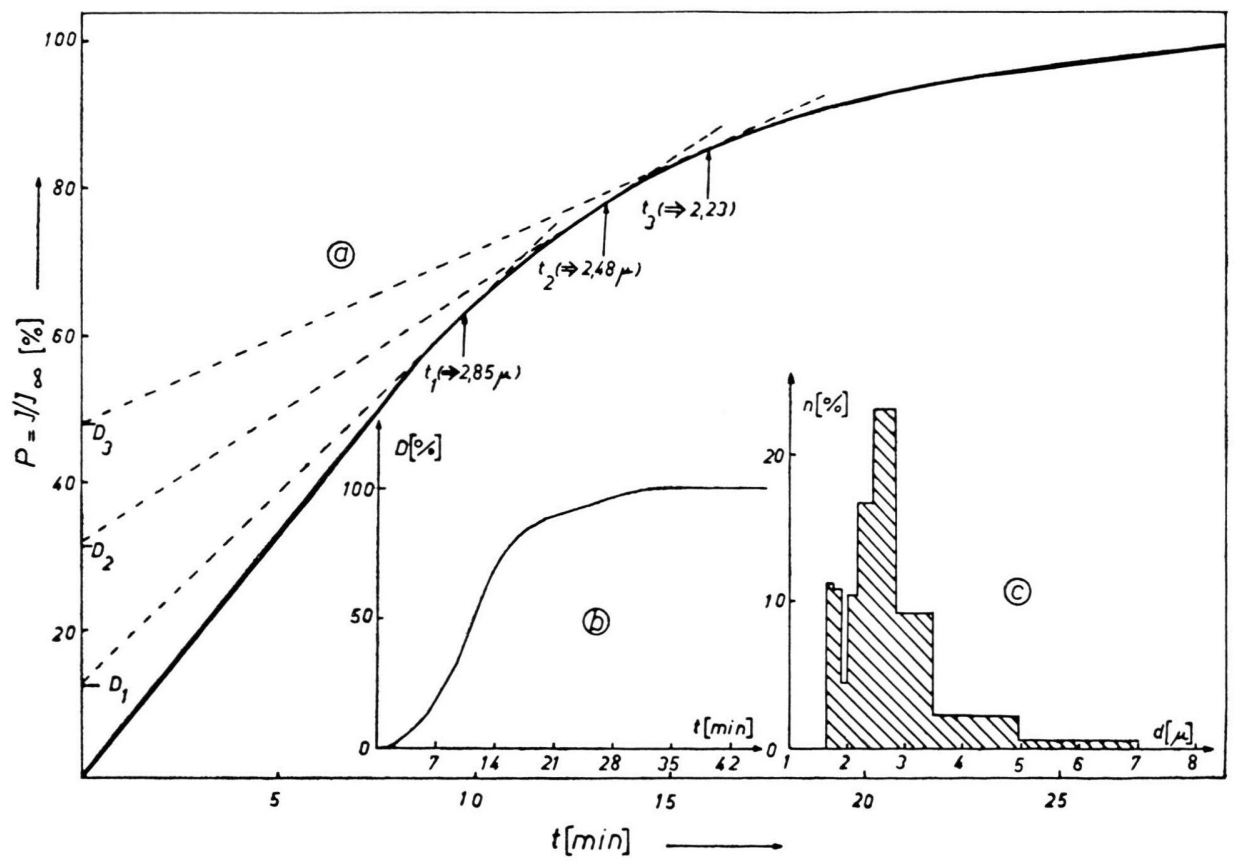

Abb. 2. Sedimentationskurve und Korngrößenverteilungskurve eines $\mathrm{UO}_{\mathbf{z}}$-Pulvers.

3 S. ODÉN, Koll.-Z. 18, 33 [1916]. 
wird die Variable $t$ gegen die Variable $r$ (Korngröße) unter Benutzung der Sтокеsschen Gleichung in der Form $d=k \sqrt{1 / t}$ ausgetauscht, was zu

$$
\frac{\mathrm{d} D(t)}{\mathrm{d} d}=2 \frac{1}{k} t^{5 / 2} \frac{\mathrm{d}^{2} P(t)}{\mathrm{d} t^{2}}
$$

führt. Unter Berücksichtigung der verschiedenen Vorzeichen $[\mathrm{d} D(t) / \mathrm{d} d \leq 0 ; F(r) \geq 0]$ besteht Übereinstimmung mit ODÉNs Massenverteilungsfunktion

$$
F(r)=-2 \sqrt{\frac{c}{h}} t^{5 / 2} \frac{\mathrm{d}^{2} P(t)}{\mathrm{d} t^{2}} .
$$

Zwei verschiedene Anwendungsbereiche der Methode können unterschieden werden:

1. Bestimmung der Teilchengröße (bzw. -Dichte) von homogen $\alpha$-radioaktiven Stoffen, wie z. B. schwerlöslichen Uranverbindungen oder durch Mischfällung mit $\alpha$-strahlenden Nukliden erhaltenen Niederschlägen oder homogen beladenen Ionenaustauschern.

2. Bestimmung an durch Oberflächenadsorption aktivierten Teilchen, wie z. B. gewissen Oxyden, Silikaten, Gläsern.

Für beide Möglichkeiten werden im folgenden charakteristische Beispiele gegeben:

1. Urandioxyd. $50 \mathrm{mg} \mathrm{UO}$ der $\mathrm{AB}$ Atomenergi, Stockholm, mit einer Korngröße von $2-4 \mu$ (gemäß Abtrennung mittels Andreasen-Pipette ${ }^{4}$ ) wurden in einem Sedimentationszylinder von $4 \mathrm{~cm}$ Höhe in $0,02 \mathrm{M} \mathrm{Na}_{2} \mathrm{P}_{2} \mathrm{O}_{7}$ Lösung (Dispergierungsmittel zur Vermeidung von Agglomeratbildung) aufgeschlämmt. Aus der in Abb. 2 wiedergegebenen Sedimentationskurve ließ sich, wie

4 A. H. M. Andreasen u. V. Lundberg, Ber. dtsch. Keram. Ges. 11, 249 [1930].

5 B. M. Abraham, H. E. Flotow u. R. D. Carlson, Analyt. Chem. 29, 1058 [1957].

\section{Isotopic Composition of Xenon from Enstatite Chondrites *}

\author{
By J. H. Reynolds \\ Miller Institute for Basic Research in Science, \\ and Department of Physics, University of California, \\ Berkeley \\ (Z. Naturforschg. 15 a, 1112-1114 [1960]; eingeg. am 28. Oktober 1960)
}

In previous letters ${ }^{1-3}$ we have reported two types of isotopic anomaly in xenon from stone meteorites. First, there is often a marked increase of $\mathrm{Xe}^{129}$, as in the ordinary chondrite Richardton ${ }^{1}$ where the $\mathrm{Xe}^{129} / \mathrm{Xe}^{132}$ ratio is 1.5 instead of 0.98 as in the atmosphere. Second, there are less obvious anomalies - which we have called

* Work supported in part by the U.S. Atomic Energy Commission.

1 J. H. Reynolds, Phys. Rev., Letters 4, 8 [1960].

2 J. H. Reynolds, Phys. Rev., Letters 4, 351 [1960].

3 J. H. Reynolds, J. Geophys. Res. 65, 3843 [1960]. oben beschrieben, die Häufigkeitsverteilungskurve ermitteln, die ebenfalls in Abb. 2 wiedergegeben ist.

2. Pyrexglaspulver. Nach Mörsern, Sieben und Fraktionierung mit der Andreasen-Pipette wurde eine Fraktion im Korngrößenbereich $2-25 \mu$ erhalten. An diesem Pulver wurde $\mathrm{Pb}-212$ (ThB) im Gleichgewicht mit Bi-212 (ThC) aus schwach alkalischer trägerfreier Lösung adsorbiert. Nach gründlichem Waschen mit destilliertem Wasser gelangten $25 \mathrm{mg}$ des Pulvers zur Sedimentation. Die Auswertung geschah wie oben, die Voraussetzung konstanter Flächenbelegung (Indizierung) wurde durch Messung der spezifischen Aktivität an verschiedenen Fraktionen bestätigt; die mittlere Korngröße wurde zu $14 \mu$ gefunden.

Ein Vorteil der beschriebenen Methode ist es, daß sehr kleine (unwägbare) Mengen für eine Bestimmung ausreichen, und daß die Durchführung in jedem radiochemischen Laboratorium sehr einfach ist, da die Apparaturteile in jedem Fall zur Verfügung stehen.

Ein Vergleich mit anderen in der Literatur beschriebenen radioaktiven Methoden zeigt folgendes: Verglichen mit der Methode der Teilchengrößenbestimmung mittels $\gamma$-Messung nach Neutronenbestrahlung ${ }^{5}$ werden im vorliegenden Fall bedeutend geringere Radioaktivitätsmengen benötigt; verglichen mit der Methode der Bestimmung mittels der Rückstreuung von $\beta$-Strahlung ${ }^{6}$ ist die vorliegende Methode auch bei den Verbindungen leichter Elemente verwendbar und bedarf keiner langwierigen Kalibrierung.

Wir danken dem Sch wedischen A tom for. s chung s r a t für Unterstützung.

- P. Connor, W. H. Hardwick u. B. J. Lanndy, J. Appl. Chem. 9, 525 [1959].

secondary anomalies - in most of the other isotopic ratios. The secondary anomalies occur in various stone meteorites and have been shown ${ }^{2}$ to be primordial and unrelated to cosmic ray exposure of the stones. The carbonaceous chondrites exhibit the secondary anomalies with great uniformity ${ }^{3}$. Since these particular stones are very rich in xenon (exceeding ordinary chondrites in xenon content by a factor of about 40 and exceeding the earth in xenon content by a factor which may be as high as 800) it is very reasonable to infer with Kuroda ${ }^{4}$ and Cameron ${ }^{5}$ that xenon from the carbonaceous chondrites is the best available sample of primordial xenon and that differences between this xenon and the xenon in the atmosphere must be largely attributed to processes which have changed the latter. It

4 P. K. Kuroda, Nature, Lond. 187, 36 [1960].

5 A. G. W. Cameron, Differences between atmospheric and meteorite xenon, Section II in the formation of the son and planets, to be published. 